\title{
Quantitative Research in Tourism and Hospitality: An Agenda for Best Practice Recommendations
}

\begin{abstract}
Purpose

This paper serves as an important guide for more rigorous quantitative research in tourism and hospitality.

\section{Design/methodology/approach}

The paper relies on comments from several methodological experts in the field, as well as the authors' main observation of the literature

\section{Findings}

The paper identifies ten important areas of concern. In each of these areas we provide recommendations for best practices.

\section{Research limitations/implications}

There are certainly other issues and concerns that are not covered in this paper. However, the issues addressed can be applied or generalized to most methodological contexts.

\section{Originality/value}

This paper does not present results from original research but provides interesting and comprehensive recommendations for more rigorous quantitative research.

\section{Introduction}

Interest in quantitative methodologies across various areas of tourism and hospitality research has increased significantly in recent years. To provide some best practice recommendations, this article identifies primary area of concern, and provides an agenda for methodological improvements. We rely on comments from several methodological experts in the field, as well as our main observation of the literature. In particular, we focus on the following issues: "building better regression models", "checking and remedying the effect multicollinearity", "properly testing for shape, main effect and moderation in curvilinear models", "assessing the predictive ability of your model", "do not abuse the p-value:, "thinking beyond conventional regression methods", "more dependence on panel data", "more dependence on the Bayesian approach for hypothesis testing and model estimation", and finally, "shifting toward better practices in structural equation modelling".

In each of these areas, the paper provides recommendations for best practices. Discussing them is in order does not prioritize one over another, but is mainly related to the flow or argumentsall these issues are somehow interrelated. The paper does not cover all issues and concerns but 
focuses mainly on those that can be applied or generalized to several methodological contexts. The goal was not to position this paper as a critique of the existing literature, but to make a list of best practice recommendations.

\section{Building Better Regression Models: Keep it Simple and Report All Diagnostics}

It is common among young researchers to think that estimating more complicated regression models leads to better outcomes. However, this is not necessarily true. It is important to keep models "sophisticatedly simple" (Zellner, 2001). Model selection should depend on the appropriate specification tests in order to ensure the selection of the appropriate functional form as well as the "correct" variables. No such thing as a "correct" model exists so, here, the term "acceptable" means that a given model has sufficient support in the light of the data, and after a set of diagnostic tests has been passed.

Unfortunately, it is rare to see tourism papers testing these issues prior to reporting the regression results. One needs to plot the residuals against the independent variables to see if there any pattern of the residual becoming larger or smaller as the independent variable increases or decreases. Some misspecification tests such as the "Ramsey Reset Test" can also detect problems with functional forms and should be regularly reported ${ }^{1}$. As emphasized by Assaf and Tsionas (2019a), "problems like autocorrelation and heteroscedasticity should be also interpreted as misspecification tests rather than as problems that merely affect standard errors (which is the case only in correctly specified models)". Hence, testing for heteroscedasticity and autocorrelation is also important. Simply using heteroscedasticity-robust standard errors (as it is common literature) should not overcome the need to report all the necessary specification and heteroscedasticity tests (Assaf and Tsionas, 2019a).

It is important to emphasize that residuals are highly important in detecting violations of model assumptions. A careful analysis of residuals is rarely reported in tourism papers or in social science research in general. These "range from inappropriate tests of the significant of coefficients (either showing significance when it is not present or vice versa) to the biased and inaccurate predictions of the independent variables" (Hair et al. 1998, p. 176). The residuals can reveal any violation of linearity, independence, homoscedasticity, and normality. Importantly, it is also essential to use the residuals to check for influential observations and outliers. Reporting, for instance, measures such deleted, standardized or student residuals can help reveal any influential observation in the data. Such observations do not necessarily need to be deleted but their impact on the results should be appropriately checked.

Correct and comprehensive reporting of the regression results is also important. Depending on the context, it may be necessary to report the effect sizes after fitting regression models. Reporting the standardized regression coefficients may not be sufficient as these can be affected by the overall correlation among the predictors in the models. Other measures such as the

\footnotetext{
${ }^{1}$ It is also important to check for omitted-variable bias. The Ramsey's test should not be perceived as test for omitted- variable bias.
} 
semipartial (part) correlation which are much more informative than the standardized regression coefficients are not often reported. Caution should be also used in terms of interpreting the standardized regression coefficients. For instance, they should be interpreted relative to other variables in the models, and not in absolute sense. In practice, reporting elasticities and standard errors may be preferable.

\section{Checking and Remedying the Effect of Multicollinearity}

Checking for multicollinearity is another important robustness check that should also accompany the building of better regression models. It is important to highlight this issue separately due to some common problems that exist in the field. The following issues, in particular, require careful consideration:

1- First, examining only the correlation matrix does not reveal the extent of multicollinearity problem. Even the variance inflation factors, commonly used in the literature, do not always reveal the extent of multicollinearity. It is recommended instead to rely on the condition index and the variance decomposition matrix to assess the extent of multicollinearity. These diagnostics are rarely reported in the literature.

2- Second, mean centering the regression variables does not address the multicollinearity problem. Recent evidence has shown that such procedure does not really work (Echambadi and Hess, 2007). While the correlation matrix may indicate that the mean centered variables have less correlation, the results (if interpreted correctly) will be actually identical to the model without mean centering. In other words, mean centering does not increase or alleviate the multicollinearity problem.

3- Third, it is important to emphasize that dropping highly correlated variables from the model does not also seem to be a good solution to address the multicollinearity problem, as shown in recent simulation evidence (Assaf et al. 2019b). Dropping variables from the model also introduces the risk of misspecifying the regression model.

To remedy the effect of multicollinearity we recommend instead relying on more sophisticated methods such as Bayesian regression, and more specifically ridge regression (Hair et al. 1998; Assaf et al. 2019b) which is, in reality, Bayesian regression with an informative prior on the coefficients. The use of Bayesian regression has not been so far highly common in the tourism literature. In a recent paper, Assaf et al. (2019b) have demonstrated the power of Bayesian ridge regression in effectively handling the multicollinearity problem, using evidence from both simulated and real datasets. It is also less well known that multicollinearity may indicate endogeneity in the sense that certain explanatory variables may be statistically related as well as related to the dependent variable of the model. In such cases, multicollinearity is a problem of specification, rather than a mere cause of inflated standard errors associated with otherwise consistently estimated parameters. We refer the reader to Assaf et al. (2019b) for more technical details. 


\section{Properly Testing for Shape, Main Effects and Moderations in Curvilinear Models}

Along with the above, we recommend more consistent and correct testing of curvilinear regression models. Despite being highly common in hospitality and tourism research, researchers in the field tend to make the following four mistakes when analysing such models.

1- The first is related to testing the shape of such models. Consider, for example, the following regression model:

$$
y=\beta_{0}+\beta_{1} x+\beta_{2} x^{2}+\varepsilon
$$

where the goal is to test whether there is a U-shaped or inverted U-shaped relationship between $x$ and $y$. A common practice when testing such hypothesis is to rely on the sign of $\beta_{2}$. When $\beta_{2}<0$, it is common to assume a $U$-shaped relationship (the opposite is true for inverted $\mathrm{U}$ ). However, such process is incomplete. To confirm a U-shaped relationship, one needs also to confirm that the slope at the low and high ends of $x$ is significant, and that the turning point of the curve is within the range of data (see Haans et al. 2016 for details). If any these steps fail, one cannot claim then to have a full Ushaped relationship. Unfortunately, testing for all these conditions is not currently common the tourism literature.

2- The second is related to testing the impact of $x$ on $y$ in a model such as (1). Most papers test the hypothesis involving " $x$ " using an additional model like ( $\left.y=\beta_{0}+\beta_{1} x+\varepsilon\right)$, also labelled a "main effect model" where the effect of $x^{2}$ has been removed from the model. Unfortunately, such procedure is potentially incorrect. When a term like $x^{2}$ is present, and if it has been theoretically supported, any hypothesis should be tested when $x^{2}$ is present in the model. This should be done by taking the derivative of this model with respect to $x$ (i.e. marginal effect), evaluated at the whole range of the data (Software packages like STATA can be used for that purpose). The reason is that the model with the squared term does not admit consistent estimation.

3- The third is related to testing for the moderating effect in a model like (1). The common practice in the field is to extend (1) using a model such as:

$y=\alpha_{0}+\alpha_{1} x+\alpha_{2} z+\alpha_{3} x^{2}+\alpha_{4} x z+\varepsilon$,

where $z$ is the moderator, and the moderating effect is tested using $\alpha_{4}$. We argue that for richer testing, studies should consider a more complete model such as:

$y=\alpha_{0}+\alpha_{1} x+\alpha_{2} z+\alpha_{3} x^{2}+\alpha_{4} x z+\alpha_{5} x^{2} z+\varepsilon$

where the moderator $z$ is now also multiplied by the non-linear term $x^{2}$. Adding the term $x^{2} z$ allows for testing of richer moderating effects. For example, one can then test for how the moderator shifts (i.e. turning point) or flattens/steepens the $U$ (or inverted 
U)-shape relationship between $x$ and $y$ (Haans et al. 2016). While papers in mainstream and marketing journals commonly test such hypotheses (Henderson et al. 2006), it is rare to see a tourism paper focusing on these issues.

4- Finally, the fourth is related to some important robustness checks that are largely ignored with models such as (1). For instance, along with the three recommendations above, we it is important to test for endogeneity as "quadratic specifications are at risk of running a particular form of forbidden regression" (Haans et al. 2016, p.1183). Ignoring such issues may lead to inconsistent estimation.

\section{Assessing the Predictive Ability of Your Model}

The focus in the field has always been on using statistical models to explain a certain causal theory. Very little focus has been placed on the predictive ability of these models. For instance, simply comparing the performance of regression or other related models based on the value of $\mathrm{R}$-squared is never sufficient. A more effective way to test the predictive ability against other models is to conduct out-of-sample validation, which means estimating the model on one part of the data, and then test the predictability ability of the model on the rest of the data also known as "hold out sample". Examining the out-of-sample performance, can reveal any deficiency in the model. For a visual representation one can also plot the deviations between actual and predicted values out-of-sample. Of course, the sizes of the two sub-samples must be selected so that we have enough observations in both the fitting and validations samples.

We believe that testing the predictive ability of the model should be always reported in future papers. Any acceptable model should provide good predictions as all science is essentially predictive in nature. Such criteria should not be limited to regression models but to any other statistical model or estimation method. The Bayesian approach, in particular, offers unique advantages in terms to assessing the predictive ability of statistical models. Given data $Y=$ $\left[y_{1}, \ldots, y_{T}\right]^{\prime}$ and parameters $\theta$, the Bayes' theorem can be used to provide the posterior distribution:

$$
p(\theta \mid Y) \propto L(\theta ; Y) p(\theta),
$$

where $L(\theta ; Y)$ is the likelihood and $p(\theta)$ is the prior. To predict the next observation we need the density $p\left(Y_{T+1} \mid Y\right)$ which is known as the posterior predictive density. This can be computed using the following:

$p\left(Y_{T+1} \mid Y\right)=\int p\left(Y_{T+1}, \theta \mid Y\right) d \theta=\int p\left(Y_{T+1} \mid \theta, Y\right) p(\theta \mid Y) d \theta$.

If a Markov Chain Monte Carlo (MCMC) sample $\left\{\theta^{(s)}, s=1, \ldots, S\right\}$ is available, the integral can be accurately approximated using:

$$
p\left(Y_{T+1} \mid Y\right) \cong S^{-1} \sum_{s=1}^{S} p\left(Y_{T+1} \mid \theta^{(s)}, Y\right),
$$


where $p\left(Y_{T+1} \mid \theta^{(s)}, Y\right)$ is often available in closed form. One way to compare forecasts is to use the log-predictive score:

$$
L P S=\log p\left(Y_{T+1} \mid Y\right) .
$$

If $p\left(Y_{T+1} \mid Y\right)$ is normal, then $-2 L P S$ provides a mean squared error, so LPS is a measure of fit that can be computed easily for most models through MCMC methods.

If we have different models indexed by $m \in\{1, \ldots, M\}$ one can compute the predictive densities and compare either the different LPSs or the ratio

$$
\frac{p_{m}\left(Y_{T+1} \mid Y\right)}{p_{1}\left(Y_{T+1} \mid Y\right)}, m=2, \ldots, M,
$$

which is the predictive Bayes factor in favor of model $\mathrm{m}$ and against model 1 . Here, $Y_{T+1}$ denotes the actual future observation (perhaps in a hold-out sample).

Several techniques for forecast combination and model comparison using the predictive densities are proposed in Geweke and Amisano $(2010,2011)$ where the reader is referred to for further details. The natural advantages of the Bayesian approach in prediction, are that i) one does not rely on asymptotic approximations, and ii) the posterior predictive density gives a full summary regarding prediction of a future observation. Of course, the analysis can be extended in a straightforward way when the objective is to predict a set of future values, say $Y_{T+1}, \ldots, Y_{T+h}$.

\section{Do Not Abuse the P-value}

To improve the replication of existing studies, there is an ongoing debate across several science fields as to whether the p-value threshold should stay at the arbitrary level of 0.05 or become even lower (Barach et al. 2018). There are also some misconceptions that exist in the field about the true meaning of $\mathrm{p}$-value. With a p-value that is lower than 0.05 , for instance, it is common to state that "we reject the null". This interpretation, however, is problematic, as "the p-value is derived with the assumption that the null is true, so how it can be also the probability that the null is false?" (Assaf et al. 2018a, p.18). In other words, "a p-value of 0.05 does not mean that there is a $95 \%$ chance that a given hypothesis is correct. Instead, it signifies that if the null hypothesis is true, and all other assumptions made are valid, there is a $5 \%$ chance of obtaining a result at least as extreme as the one observed" (Baker, 2016, p.531).

The American Statistical Association (ASA) has recently released a statement to clarify the above (Wasserstein and Lazar, 2016), and provided several recommendations on the proper use and interpretation of $\mathrm{p}$-values. Studies in the field should not necessarily stop using p-values for hypothesis testing, but it is important to avoid its common misinterpretation (Amrhein et al. 2019). Obtaining a significant result is still rewarding, but one has to understand what this really represents. In line with the recommendation of ASA, we suggest the following practices for studies in the field: 
1- Do not use p-values to interpret the size of the effect or the importance of the results. Even small effects can produce small p-values when the sample size is large.

2- Report all inferences including both small and large p-values. In other words, do not selectively report significant findings.

3- Report the exact p-values. For instance a p-value of 0.05 is also not the same as $\mathrm{p}<0.05$.

4- Try not to base your conclusions solely on a small $\mathrm{p}$-value but provide evidence from other methods and approaches.

Recent papers have recommend using the Bayes factors as an alternative to p-values (Dienes and Mclatchie, 2018). The Bayes factor "relies only on the observed data at hand, and not on some hypothetical repeated samples, which we do not observe and they are the essence of the calculation of the p-value" (Assaf and Tsionas, 2018a, p.21). In contrast to p-values, the Bayes factor accounts for the likelihood and prior evidence under both $\mathrm{H}_{0}$ and $\mathrm{H}_{1}$. Finally, the Bayes factor reflects the size of an effect. We refer the reader to Assaf and Tsionas (2018a) for a detailed discussion on the concepts of $\mathrm{p}$-values and Bayes factors. The authors also provide detailed evidence about the performance of the two in different datasets. In general, they demonstrated that the Bayes factors is less sensitive and does not fluctuate from sample to sample as much as the p-value.

\section{Thinking beyond Conventional Regression Methods}

The use of common regression methods such as linear regression has always been the norm in the field. Other types of regression models such as Quantile regression and Non-Parametric regression have recently started to gain some attention in the literature (Hung et al. 2010; Lew and $\mathrm{Ng}$, 2012; Masiero et al. 2015). We strongly recommend more use of these methods as they provide several important advantages and robustness to the findings. The conventional regression methods, for instance, only allow estimation of the average relation between the covariates and the outcome variable, while quantile regressions assess the covariate effects at different quantiles of the outcome variable (Li, 2015; Assaf and Tsionas, 2018b). Hence, they provide a more complete picture and richer information for hypothesis testing. In areas like tourism where many empirical applications involve heavy tailed distribution, quantile regressions can bring an additional advantage.

Non-parametric regressions (NPRs) have also been less popular in tourism research. These models should be more commonly used as they free the researcher from the need to pre-assign a functional form between the outcome and predictor variables, and are less subject to specification problems. NPRs can also be highly useful in moderated regression models where one can uncover the full moderating effect instead of simply taking the linear interaction of the moderator and predictor variables (Assaf and Tsionas, 2019c). 


\section{More Dependence on Panel Data}

The use of panel data for the estimation of regression and other related models is also always encouraged. By panel or longitudinal data, we mean observations that are repeated over time across cross-sectional units such as individuals, firms, and countries. Obvious advantages of panel data are the more accurate and easier estimation and inferences of model parameters, given the higher degrees of freedom and sample variability. Panel models are also better equipped to control for unobserved heterogeneity and omitted variable bias. With larger data, one can also test for complicated behavioural hypotheses. Finally, panel models can uncover some interesting dynamic relationships in the data.

Hence, we encourage the use of panel data not only for econometric models but also in other contexts such as structural equation modelling (SEM). The two most common specifications of panel data are the fixed effects (FE) and random effects (RE) specifications and each brings different types of advantages. With the random effects, one can obtain more efficient estimators and include time invariant variable in the estimation. Additionally, the number of parameters does not increase when the number of observations increases. The fixed effects specification has the advantages of allowing the individual or time effect to correlate with the predictor variables. Hence, it is much less restrictive than the random effects. The disadvantage, however, it that it does not allow for time invariant variables. The number of unknown parameters also increases with the number of observations. Of course, it is also affected by the incidental parameter problem.

Both FE and RE have been common in tourism research, and the selection between the two has also always been a source of debate. The Hausman test has been traditionally used to decide on which specification fits the data better. As the Hausman test, however, has its limitations one can also report the results from both FE and RE specifications. The Lagrange multiplier test can also be used to decide between a random effects regression and a simple least square regression. We also recommend consistent checking of autocorrelation, heteroscedasticity and reverse causality. Future applications may also consider more use of dynamic panel models (Flannery and Hankins, 2013). It is unfortunate that most panel applications have been static in nature. Dynamic models allow the dependent variable to depend on its past realization and can prove to be very useful in many tourism and hospitality application. For instance, in applications where firm performance is used as a dependent variable, one would assume that performance is affected by its past realization as firms learn how to improve their performance over time. By being limited to static models, studies are failing to control for these important behavioural specifications. Dynamic models may also be more effective in dealing with endogeneity, simultaneity, reverse causality, omitted variable bias, and country-specific effects. 


\section{More Dependence on the Bayesian Approach for Hypothesis Testing and Model Estimation}

The use of Bayesian inference for model estimation is rapidly growing across several fields including management, marketing, psychology and tourism, as is evidenced by the recent publications and special issues on the topic (Zyphur and Oswald, 2013). The method does not simply add more flexibility to model estimation, but is a completely new paradigm that deserves special attention. So far, the sampling theory approach to model estimation (e.g. Maximum Likelihood (ML), Ordinary Least squares (OLS), etc.) has been the most common in tourism research. It is not until recently that some papers have called for more use of the Bayesian approach across several tourism fields such as performance modelling (Assaf and Tsionas, 2017), structural equation modelling (Assaf et al. 2018c) and forecasting (Wong et al. 2006; Assaf et al. 2018d).

The Bayesian approach introduces several advantages including "rich diagnostic information about parameters and models; controlling for multiple comparisons as a function of the data; handling low-frequency, unbalanced, missing data; and exploration of prior assumptions about model parameters" (Zyphur and Oswald, 2013, p.7). Above, we also discussed how the Bayesian approach is more effective with collinear data. Probably, one of the known advantages of the Bayesian approach is that it "makes direct probability statements about the parameters using the observed sample". The p-value for example is derived based on the assumption of drawing from an infinite number of samples, which we do not really observe. Moreover, the Bayesian approach works better in finite samples, and "has the advantage of incorporating prior information (about previous findings and theory) into the estimation, which sometimes can prove to be highly useful” (Assaf et al., 2018c, p.100).

All these advantages translate to the concept of Bayes factor as we discussed above. The Bayesian approach also offers other tools for hypothesis that can be used in tourism literature, and have better properties than the p-value. For example, one can use the $95 \%$ higher posterior density (HPD) interval to test the significance of a certain effect. Like the confidence interval, the HPD interval can also inform us about the "magnitude of sampling variability". However, the HPD interval has nicer properties compared to traditional confidence intervals. We refer the reader to Assaf and Tsionas (2018a) for more details. Several papers have also discussed the power of the Bayesian approach within specific modelling frameworks such as structural equation modelling (Muthén and Asparouhov, 2012), performance modelling (Assaf et al. 2017), and forecasting (Wong et al. 2006). Hence, these issues will not be reiterated here.

\section{Shifting toward better Practices in Structural Equation Modelling}

All nine issues we discussed above equally apply to SEM, which continues to be one of the most popular and particularly useful multivariate analysis methods in the field. At the same time, many studies show serious problems in the model estimation and results evaluation. Oftentimes, this is not merely a problem with the method but the researchers who simply abuse the tool for their purposes (Petter 2018). For this reason, we like to highlight on some "best practice 
recommendations" in order to ensure more the rigor, consistency, and transparency in future research. We divide this section into three parts. First, we provide recommendations in terms of reporting the SEM results. Second, we focus on the importance of method selection, in particular the difference between covariance-based SEM (CB-SEM) and partial least squares SEM (PLS-SEM). Finally, we discuss the use of Bayesian SEM

\subsection{Reporting the SEM Results}

In terms of reporting the SEM results, and based on the current inconsistencies we have in the tourism literature, we believe that all papers should:

1- Report all the number of observations needed given the model under analysis.

2- Provide graphical illustration for both measurement and structural models.

3- Check and report any violation of univariate and multivariate normality for all measured indicators.

4- Check and report any violation of other important assumptions such as linearity and independence.

5- Provide the covariance matrix of all observed variables (or the correlation matrix along with the standard deviation).

6- Highlight the proportion of missing data (if any) and the actions you took to address this problem.

7- Indicate the method of estimation.

8- Discuss which fit indices (overall, absolute, and incremental) - if applicable - have been selected and provide justifications for selecting them.

9- Report all parameter estimates including variances, standard errors, p-values, $R^{2}$, standardized and unstandardized structure coefficients, and clearly highlight any path that has been fixed.

10- Report the indicator and composite reliabilities, the average variance extracted (AVE), and the discriminant validity of the construct measures.

11- Test and report the results of cross validation on a hold-out sample.

12- Provide results from at least one competitive model (Sharma et al. 2019). It is an open problem what "competitive" models one should consider and how robustness of certain estimates can be established. Relatedly, researchers should also consider checking for any unobserved heterogeneity in the data (Assaf et al. 2016; Sarstedt et al. 2010)

13- State the software used to estimate the data.

\subsection{CB-SEM vs. PLS-SEM}

In a recent paper, Rigdon et al. (2017) provided a very comprehensive summary of the different perspectives when comparing CB-SEM and PLS-SEM. First, we fully agree with their statement that the focus should be primarily on the quality of the research process and the data and not on the never-ending argument and comparison between two methods that are fundamentally very different in the first place. We also believe that both methods have their strengths and weaknesses, and we reinforce the argument that CB-SEM and PLS-SEM serve two different purposes. PLS-SEM is more prediction-oriented and can better handle complex models, small 
samples, and formative constructs (Sarstedt et al. 2017). CB-SEM on the other hand is more suited for theory testing. Hence, the selection between the two should be based on the research purpose at hand, and "instead of seeking confidence in the comparison of results from the different approaches, researchers should instead focus on more fundamental aspects of modeling, measurement, and statistical analysis" (Rigdon et al. 2017, p. 7). In addition, while CBSEM has the strength of providing goodness-of-fit criterion, the focus should not be on solely reaching a good fit at the expense of making fundamental change to the research design or theoretical arguments. Otherwise, the outcome will be a "best-case scenario that almost never applies in reality" (Sarstedt et al. 2014, p. 157).

\subsection{Bayesian SEM}

On a final note, we also recommend more use of Bayesian SEM, which shares many characteristics of the two methods highlighted above, and introduces several other advantages. The Bayesian approach, for instance, can better handle more "complicated data structure and model assumptions", and introduces prior information into the analysis. It also offers more accurate fit indices and model comparison criteria, and can easily assess the predictive ability of SEM model. Finally, the Bayesian approach is more robust to small sizes. We refer the reader to Assaf et al. (2018c) for technical details and a detailed overview of Bayesian SEM.

\section{Final Remarks}

The purpose of this paper was to provide an agenda for future methodological improvements in tourism and hospitality research. We focused and provided recommendations on ten different issues. We relied on our own observation of the literature as well as the comments of several methodological experts in the field. To sum up, we recommend more focus on the areas highlighted above. In each of these areas, our recommendations for best practices can be generalized to most modelling contexts. Finally, it is important to emphasize that most of these recommendations will be not effective (regardless of the methodology or method of estimation) if we do not have representative samples. Often, researchers just collect data without ensuring the representativeness of their sample. Sometimes, they even have only a vague idea of the population. This issue (representativeness of the sample) is often (usually) neglected in publications. A non-representative sample is a major threat to the validity of results. In addition, missing values represent an important issue. Researchers are encouraged to be more conservative with missing values (e.g., only accept levels of $10 \%$ or less) and should always highlight the missing value treatment option used. 


\section{References}

Amrhein, V., Greenland, S., \& McShane, B. (2019). Scientists rise up against statistical significance. Nature 567, 305-307 (2019).

Assaf, A. G., Oh, H., \& Tsionas, M. (2017). Bayesian approach for the measurement of tourism performance: A case of stochastic frontier models. Journal of Travel Research, 56(2), 172-186.

Assaf, A. G., \& Tsionas, M. (2018a). Bayes factors vs. P-values. Tourism Management, 67, 17-31.

Assaf, A. G., \& Tsionas, M. (2018b). Changing The Basics: Toward More Use of Quantile Regressions in Hospitality and Tourism Research. International Journal of Hospitality Management, 72, 140-144.

Assaf, A. G., Tsionas, M., \& Oh, H. (2018c). The time has come: Toward Bayesian SEM estimation in tourism research. Tourism Management, 64, 98-109.

Assaf, A. G., Li, G., Song, H., \& Tsionas, M. G. (2018d). Modeling and Forecasting Regional Tourism Demand Using the Bayesian Global Vector Autoregressive (BGVAR) Model. Journal of Travel Research, in press.

Assaf, A. G., \& Tsionas, M. G. (2019a). On proper specification in tourism research. Annals of Tourism Research, in press.

Assaf, A. G., Tsionas, M., \& Tasiopoulos, A. (2019b). Diagnosing and correcting the effects of multicollinearity: Bayesian implications of ridge regression. Tourism Management, 71, 1-8.

Assaf, A. G., \& Tsionas, M. (2019). Non-parametric regression for hypothesis testing in hospitality and tourism research. International Journal of Hospitality Management, 76, 43-47.

Assaf, A. G., Oh, H., \& Tsionas, M. G. (2016). Unobserved heterogeneity in hospitality and tourism research. Journal of Travel Research, 55(6), 774-788.

Baker, M. (2016). Statisticians issue warning over misuse of P values. Nature News, 531(7593), 151.

Barach, P., Thomas, R. L., \& Lipshultz, S. E. (2018). Lowering the P Value Threshold. JAMA, 320(9), 936-936.

Dienes, Z., \& Mclatchie, N. (2018). Four reasons to prefer Bayesian analyses over significance testing. Psychonomic bulletin \&o review, 25(1), 207-218.

Echambadi, R., \& Hess, J. D. (2007). Mean-centering does not alleviate collinearity problems in moderated multiple regression models. Marketing Science, 26(3), 438-445.

Gefen, D., Straub, D. W., \& Rigdon, E. E. (2011). An update and extension to SEM guidelines for admnistrative and social science research. Manag. Inf. Syst. Q, 35(2).

Geweke, J., \& Amisano, G. (2010). Comparing and evaluating Bayesian predictive distributions of asset returns. International Journal of Forecasting, 26(2), 216-230. 
Geweke, J., \& Amisano, G. (2011). Optimal prediction pools. Journal of Econometrics, 164(1), 130141.

Haans, R. F., Pieters, C., \& He, Z. L. (2016). Thinking about U: Theorizing and testing U-and inverted U-shaped relationships in strategy research. Strategic Management Journal, 37(7), 11771195.

Hair, Joseph F., Rolph E. Anderson, Ronald L. Tatham, and William C. Black. "Multivariate data analysis. 1998." Upper Saddle River (1998).

Henderson, A. D., Miller, D., \& Hambrick, D. C. (2006). How quickly do CEOs become obsolete? Industry dynamism, CEO tenure, and company performance. Strategic Management Journal, 27(5), 447-460.

Hung, W. T., Shang, J. K., \& Wang, F. C. (2010). Pricing determinants in the hotel industry: Quantile regression analysis. International Journal of Hospitality Management, 29(3), 378-384.

Li, M. (2015). Moving beyond the linear regression model: Advantages of the quantile regression model. Journal of management, 41(1), 71-98.

Lew, A. A., \& Ng, P. T. (2012). Using quantile regression to understand visitor spending. Journal of Travel Research, 51(3), 278-288.

Masiero, L., Nicolau, J. L., \& Law, R. (2015). A demand-driven analysis of tourist accommodation price: A quantile regression of room bookings. International Journal of Hospitality Management, 50, 1-8.

Muthén, B., \& Asparouhov, T. (2012). Bayesian structural equation modeling: a more flexible representation of substantive theory. Psychological methods, 17(3), 313.

Petter, S. (2018). Haters Gonna Hate: PLS and Information Systems Research. ACM SIGMIS Database: the DATABASE for Advances in Information Systems, 49(1), 10-13.

Rigdon, E. E., Sarstedt, M., \& Ringle, C. M. (2017). On comparing results from CB-SEM and PLS-SEM: Five perspectives and five recommendations. Marketing ZFP, 39(3), 4-16.

Sarstedt, M., Ringle, C. M., Henseler, J., \& Hair, J. F. (2014). On the emancipation of PLS-SEM: A commentary on Rigdon (2012). Long range planning, 47(3), 154-160.

Sarstedt, M., Ringle, C. M., \& Hair, J. F. (2017). Partial least squares structural equation modeling. In Handbook of market research (pp. 1-40). Springer International Publishing.

Sharma, P. N., Sarstedt, M., Shmueli, G., Kim, K. H., \& Thiele, K. O. (2019). PLS-based model selection: the role of alternative explanations in information systems research. Journal of the Association for Information Systems (forthcoming).

Wasserstein, R. L., \& Lazar, N. A. (2016). The ASA's statement on p-values: context, process, and purpose. The American Statistician, 70(2), 129-133. 
Wong, K. K., Song, H., \& Chon, K. S. (2006). Bayesian models for tourism demand forecasting. Tourism Management, 27(5), 773-780.

Zellner, A. (2001). Keep it sophisticatedly simple. In Keuzenkamp, H. \& McAleer, M. Eds. Simplicity, Inference, and Modelling: Keeping it Sophisticatedly Simple. Cambridge University Press, Cambridge.

Zyphur, M. J., \& Oswald, F. L. (2013). Bayesian probability and statistics in management research: A new horizon. Journal of Management, 39(1), 5-13. 